\title{
HF Communication During Ionospheric Storms
}

\author{
Geoffrey E. Hill
}

\section{Contribution from Research and Advanced Development Division, AVCO Corporation, Wilmington, Mass.}

(Received July 26, 1962)

\begin{abstract}
A hypothetical communication network is set up to study systematically the problem of $\mathrm{HF}$ communications during disturbed ionospheric conditions. Vertical incidence radio data is used as the basis for determining the condition of the ionosphere. Frequencies and links available have been computed for a period of severe ionospheric disturbance. Important spatial and temporal variations are clearly evident by this analysis. A comparison of the results with recorded WWV reception indicates that ionospheric vertical incidence data can be used to determine propagation conditions during disturbed periods.
\end{abstract}

\section{Introduction}

At present, HF radio communication between middle and high latitude transmitting and receiving stations is rendered ineffective during ionospheric storms. In most instances there is little or no attempt to avoid absorbing regions or to utilize anomalous reflecting regions in any systematic or knowledgeable way. It is tacitly assumed that all communication links are simultaneously affected in a more or less like manner. However, analysis of ionospheric data for several storms has shown that the spatial locations of the absorbing and reflecting lavers vary in a coherent but complex pattern throughout the lifetime of a storm.

The problem, then, is to determine practical ways by which the communication difficulties associated with ionospheric storms can be overcome, or in other words, to determine what is the optimum way in which the disturbed jonosphere should be used for a given system of transmitters and receivers. Also, it may be necessary to make alterations of an existing system so that the optimum operating procedure will lead to the reliability desired. That is, for example, the optimum operating procedure of a network of four stations is likely to give a significantly higher overall reliability than there would be with only three stations. 'Thus, the problem should be studied in such a way that improvements to a given system may be found.

To study this problem systematically, an idealized network of transmitters and receivers is introduced. It will be possible to find, or approximate, the optimum operating procedure for an ideal network. Then, when the optimum operating procedure is found for an existing system, its "effectiveness" in terms of the ideal system will be known. Recommendations for modification will be greatly facilitated by this method of study.

\section{Computations}

Before proceeding to an example, it will be helpful to show how the available frequencies and links are established. First, consider four points along a line separated by $1200 \mathrm{~km}$. A transmitter operates at one end and there are receivers at each of the remaining positions. At the receiver nearest to the transmitter we consider the arrival of a one-hop $E$ or $E_{s}$ signal, at the next, a one-hop $F_{1}$ or $F_{2}$ signal, and at the farthest, an M-hop signal. Multiple hops or combinations of different modes will be considered later.

In figure 1 the $E, F$, and $M$ modes are shown along with the required ionospheric parameters. In the case of an $E$ hop to be received at the first receiver, measurements of both the $E$ or $E_{s}$ critical frequency and absorption, using $f_{\mathrm{min}}$, are taken midway between the transmitter and receiver, i.e., midway between two neighboring points in the ideal network. In the case of an $F$-layer hop, the $f_{0} F_{2}$ is measured at the middle point where reflection occurs, and absorption is measured midway between the transmitter and the middle point for the upgoing wave and midway between the middle point and the receiver for the downcoming wave.

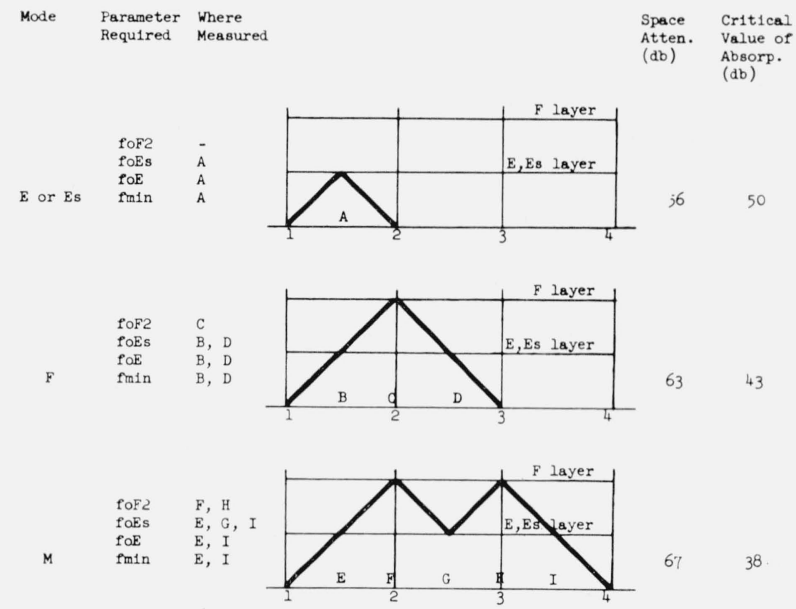

Figure 1. Schematic diagrams of modes and ionospheric parameters required to determine the frequency range applicable. The space attenuation and critical values of absorption refer to an example given in the text. 
To determine what reception is available, we will assume the signals are being transmitted at a power of $10 \mathrm{kw}$. This corresponds to about $115 \mathrm{db}$ above $1 \mu \mathrm{v} / \mathrm{m}$ at $1 \mathrm{~km}$ from the transmitter, [Rawer, 1956]. If man-made noise is neglected and atmospheric noise is assumed to be at a level of $0 \mathrm{db}$, then 115 $\mathrm{db}$ are available for the various losses encountered between the transmitter and receiver. However, the signal-to-noise ratio at the receiver must be above a certain value for adequate reception. It is assumed that this ratio is 10 , so instead of $115 \mathrm{db}$, the available amount is reduced to $105 \mathrm{db}$. About $60 \mathrm{db}$ will be lost in space attenuation. The exact amount will, of course, depend upon the particular mode. In determining whether or not the remaining $45 \mathrm{db}$ is lost, the most important factor is absorption. ${ }^{1}$ For purposes of this study it is supposed that if absorption of a signal remains less than a critical value of around $45 \mathrm{db}$ it will be detectable at the receiver. The actual critical values of absorption and amount of space attenuation for each mode are shown in figure 1 .

To understand how absorption is calculated by the use of $f_{\mathrm{m} \text { in }}$, we first consider the expression for nondeviative absorption. The signal loss, expressed in decibels, is given by

$$
L=8.7 p \sec \phi \int k d s
$$

where $p=$ number of penetrations through the $D$ region and sec $\phi=$ incidence angle of radio wave to ionosphere with

$$
k=\frac{2 \pi e^{2}}{m c \mu}\left(\frac{N \nu}{\nu^{2}+4 \pi^{2}\left(f \pm f_{L} \cos \theta f\right)^{2}}\right)
$$

where

$$
\begin{aligned}
e & =\text { charge of an electron, } \\
N & =\text { electron-number density, } \\
\nu & =\text { electron-collision frequency } \\
m & =\text { mass of an electron, } \\
c & =\text { velocity of light } \\
\mu & =\text { refractive index, } \\
f_{L} & =\text { gyrofrequency, } \\
\theta & =\text { angle between direction of propagation and } \\
& \text { magnetic field, and } \\
f & =\text { wave frequency. }^{2}
\end{aligned}
$$

For vertical incidence soundings, $p=2$, sec $\phi=1$, and $f$ is set equal to $f_{\mathrm{min}}$. $L$ at this frequency is found from the fact that at $f_{\mathrm{m} \text { in }}$ the received signal strength is just sufficient for detection by an

\footnotetext{
${ }^{1}$ Losses are assumed not to take place during ionospheric reflection except in
the case when sporadic $E$ is involved. Ground losses only occur when multiple the case when sporadic $E$ is involved. Ground losses only occur when multiple
hops or combinations are considered. Focusing and defocusing resulting from irregularities or waves in the ionosphere have been neglected.

2 The positive and negative signs in the denominator of (2) correspond to the ordinary and extraordinary waves, respectively. The electron collision frequency in the lower $D$ region (from 60 to $70 \mathrm{~km}$ ) is of the same order of magnitude as $2 \pi f$ if $f<5 \mathrm{Mc} / \mathrm{s}$, so that $\nu^{2}$ in the denominator of $(2)$ must not be neglected in determining the integral of $k d s$. For nondeviative absorption, the refractive index, $\mu$, is approximately equal to unity. For the computations, the value of $f_{L} \cos \theta=0.3 \mathrm{Me} / \mathrm{s}$ was used.
}

ionosonde, or, in other words, the absorption is at the critical value for the ionosonde. For a C-4 this value $(L)$ is about $50 \mathrm{db}$.

So eq (1) becomes

$$
50=0.92 \int \frac{N \nu}{\nu^{2}+4 \pi^{2}\left(f_{\min }+0.3 \times 10^{6}\right)^{2}} d s
$$

where

$$
\begin{aligned}
f_{L} \cos \theta & =1.5 \mathrm{Mc} / \mathrm{s}, \\
f & =f_{\mathrm{min}}, \\
L & =50 \mathrm{db} . \\
p & =2, \\
\mu & =1, \\
\sec \phi & =1, \\
2 \pi e^{2} / m c & =5.3 \times 10^{-2}(\mathrm{cgs}) .
\end{aligned}
$$

In this equation the electron-density, $N$, is still unspecified even though $f_{\mathrm{m} \text { in }}$ is specified and the electron-collision frequency, $\nu$, is approximately a fixed function of height. Of course, $N$ must be such that eq (3) is satisfied. This may be accomplished for each value of $f_{\mathrm{m} \text { in }}$ by a whole family of electrondensity height distributions. However, particular electron-density height distributions, listed in table 1 ,

\begin{tabular}{|c|c|c|c|c|c|}
\hline \multirow{2}{*}{ Height } & \multicolumn{5}{|c|}{ Distribution $\left(\mathrm{el} / \mathrm{cm}^{3}\right)$} \\
\hline & A & $\mathrm{B}$ & $\mathrm{C}$ & $\mathrm{D}$ & $\mathrm{E}$ \\
\hline $\begin{array}{c}k m \\
90 \\
80 \\
70 \\
60\end{array}$ & $\begin{array}{r}1.5 \times 10^{3} \\
10^{3} \\
2 \times 10^{2} \\
0\end{array}$ & $\begin{array}{r}1.5 \times 10^{3} \\
2 \times 10^{3} \\
1 \times 10^{3} \\
20\end{array}$ & $\begin{array}{r}1.5 \times 10^{4} \\
5 \times 10^{3} \\
6 \times 10^{2} \\
3 \times 10^{2}\end{array}$ & $\begin{array}{r}1.5 \times 10^{4} \\
2 \times 10^{4} \\
6 \times 10^{3} \\
1 \times 10^{3}\end{array}$ & $\begin{array}{r}7 \times 10^{4} \\
6 \times 10^{4} \\
1.5 \times 10^{4} \\
2 \times 10^{3}\end{array}$ \\
\hline
\end{tabular}
have been given by several workers for various ionospheric conditions [Bailey, 1959; Nicolet and Aikin, 1960; and Kane, 1960]. Although there are many other distributions leading to the same $f_{\mathrm{min}}$, the set used here is considered to be representative of real conditions.

Returning to eq (1), the amount of absorption as a function of frequency is computed for each of these electron-density distributions, and from eq (3) the corresponding value of $f_{\mathrm{m} \text { in }}$ is found. The results are shown in figure 2. Similar curves have been derived for a signal passing through the $D$ region obliquely and only once, i.e., $p=1$ and sec $\theta=5$. By interpolation of these curves the amount of absorption as a function of frequency for values of $f_{\mathrm{m} \text { in }}$ in steps of one Mc/s has been found. These results are shown in figure 3.

TABLE 1. Particular electron-density height distributions for various ionospheric conditions

Source: A and C, Nicolet and Aikin; B, D, and E, Bailey.

With the aid of figure 3 it is possible to determine the amount of absorption at any frequency by simply finding the value of $f_{\mathrm{m} \mathrm{In}_{\mathrm{n}}}$ at the places of signal passage through the $D$ region. For example, consider a $10 \mathrm{Mc} / \mathrm{s}$ signal propagated by the $F$ mode with sec $\phi=5$ at the $D$ region penetration points, $B$ and $D$, as shown in figure 1 . If $f_{\min }$ is $1.5 \mathrm{Mc} / \mathrm{s}$ at $B$ and $3.0 \mathrm{Mc} / \mathrm{s}$ at $D$, then from figure 3 it is seen that the 


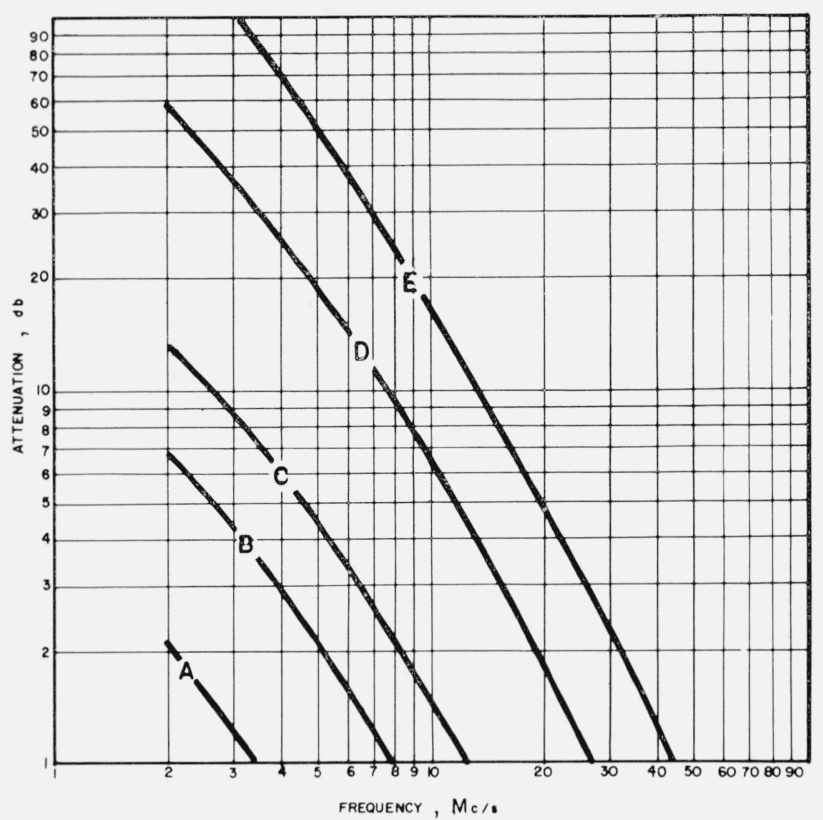

Figure 2. Amount of absorption as a function of frequency for vertical incidence using particular electron density distributions. (See table 1.) Each distribution has a corresponding value of $f_{\min }: A,<1.0 ; B$, $1.0 ; C, 1.6 ; D, 4.0$; and $E, 7.6$.

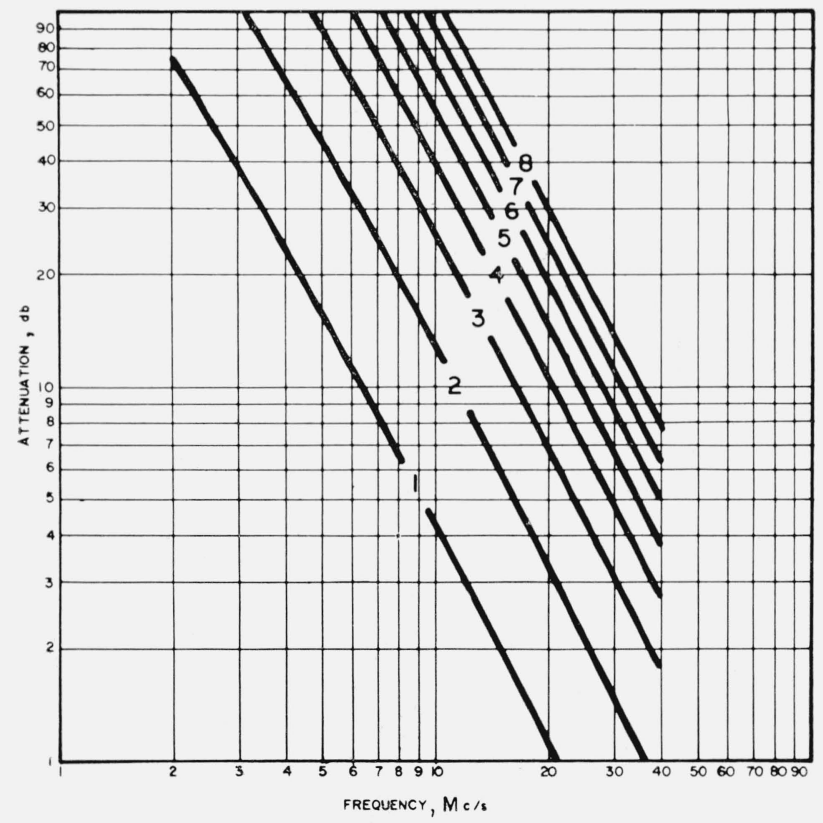

Figure 3. Amount of absorption as a function of frequency for oblique incidence ( $\sec \phi=5$ ) using the particular electron density distributions shown in figure 2.

From the values of $f_{\min }$ associated with the electron density distributions, $A$ through $E$, new curves of absorption versus frequency for oblique incidence are found for values of $f_{\mathrm{m}}$ in in steps of one $\mathrm{Mc} / \mathrm{s}$ by interpolation.

absorption is 7 and $24 \mathrm{db}$, respectively, making a total of $31 \mathrm{db}$. This value is well below the critical value of $43 \mathrm{db}$ from figure 1 for reception of a $10 \mathrm{kw}$ signal. In fact, a $1 \mathrm{kw}$ signal would also be of sufficient strength for reception.

\section{Network and Application}

The idealized network used in this study is formed by an array of equilateral triangles with a tran smitter and a receiver located at the vertices. At an interior point of the network there are six directions from which signals may arrive or depart. Each point is a distance of $1200 \mathrm{~km}$ from the neighboring ones, so that a one-hop signal covers $1200 \mathrm{~km}$ via an $E$ mode, $2400 \mathrm{~km}$ via an $F$ mode, and $3600 \mathrm{~km}$ via an $M$ mode.

To determine the frequency range and modes available over the network, values of ionospheric param. eters are taken from the appropriate points as indicated in figure 1. Then, at a given frequency, the modes possible are found from the critical frequency of the various layers and the signal's angle of incidence. Whether the resulting modes can actually be realized depends upon whether absorption is below the critical value. ${ }^{3}$

Sample tests have been made for the period of September 11-15, 1957, during which time a severe ionospheric storm took place. First, maps of the ionospheric parameters at intervals of three hours were drawn. Then, with the aid of these, communication maps were prepared for transmission frequencies of $5,10,15,20$, and $25 \mathrm{Mc} / \mathrm{s}$. For purposes of illustration only, selected communication charts are shown. These are for 10 and $20 \mathrm{Mc} / \mathrm{s}$. With these charts it is still possible to show the main effects of the ionospheric disturbance on communication. The various modes or lack of modes are indicated on these charts according to the diagram in figure 4 .

In figure 5 the communication and ionospheric charts for 1200 UT September 12 are shown. At this time the sun is illuminating the portion of the ionosphere east of the curve (on the communication charts) running through North America. Elsewhere the ionosphere is in darkness. In general, conditions in the ionosphere at this time are about normal except for polar cap absorption. The ionospheric charts are shown so that the reader may visualize the condition of the ionosphere. The curves on these charts connect points of equal values in units of $\mathrm{Mc} / \mathrm{s}$. Notice that on the $f_{a} E_{s}$ charts there are

3 For simplification it is assumed that the transmitter power, receiver gain, the minimum detectable signal-to-noise ratio, and radio noise are all constant in time, location, and frequency.

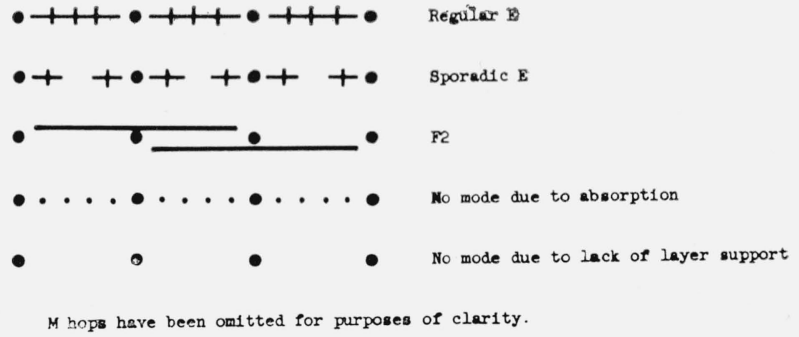

FIGURE 4. Representation of communication conditions at a fixed frequency. 
number of curves parallel to the sunrise-sunset line shown on the communication charts. These represent the regular E-layer critical frequency.

In accordance with the condition of the ionosphere, communication is about normal. On $10 \mathrm{Mc} / \mathrm{s} E$ modes prevail in the east, and $F$ and $E_{s}$ modes in the west. ${ }^{4}$ In the far north absorption is sufficient to cause outage on several links. South of Alaska there is another small region in which there is outage. This is not due to absorption, but to lack of $F$ layer support.

On $20 \mathrm{Mc} / \mathrm{s} F$ modes prevail in the east. In the west there are no available modes due to a lack of layer support. There are a few links where sporadic- $E$ support is available both in the sunlit and darkened portion of the network, but mostly in northern latitudes. No outage due to absorption is found on the $20 \mathrm{Mc} / \mathrm{s}$ chart.

To summarize, communication in the sunlit portion of the network is accomplished by E-layer support on $10 \mathrm{Mc} / \mathrm{s}$ and $F$ layer support on $20 \mathrm{Mc} / \mathrm{s}$. In the darkened portion communication is supported by the $F$ and $E_{s}$ layers on $10 \mathrm{Mc} / \mathrm{s}$, but is not supported by any layer on $20 \mathrm{Mc} / \mathrm{s}$. Some absorption on $10 \mathrm{Mc} / \mathrm{s}$ is found in polar regions. Finally, it should be mentioned that if the two charts are considered together, there are no points which are inaccessible to any other point. This condition is to be expected because the ionosphere is only slightly disturbed at this time.

4 The presence of both $F$ and $E_{8}$ modes indicates that $M$ modes are also present, although they are not indicated on the charts.

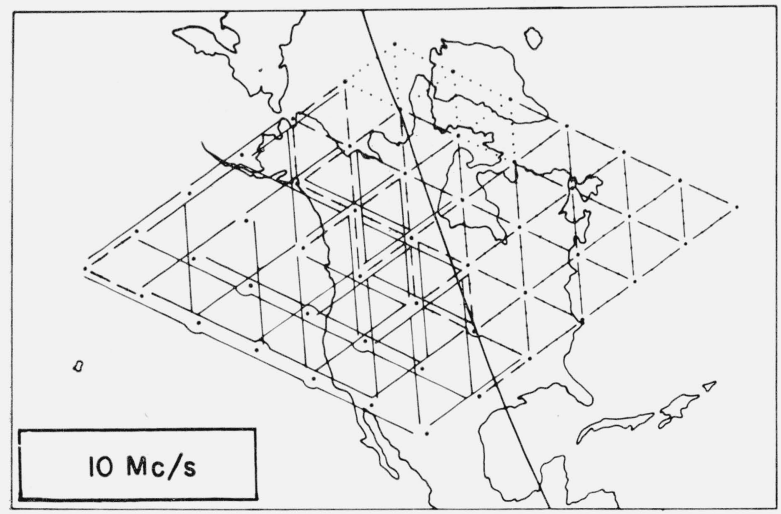

In figure 6 the charts for 0000 UT September 13 are shown. While the $F$ layer is found to be about normal, polar cap absorption is widespread and intense at this time. The communication condition is different from the previous one in two important ways. First, the illuminated portion of the network is in the west rather than the east. So, on $10 \mathrm{Mc} / \mathrm{s}$ $E$ modes are found in the west and $F$ and $E_{s}$ modes in the east. On $20 \mathrm{Mc} / \mathrm{s} F$ modes are found in the west. However, $F$ modes are also found in the east because the $F_{2}$-layer critical frequency is still high just after sunset. The second difference is that absorption in polar regions is more widespread and more intense than before. So on $10 \mathrm{Mc} / \mathrm{s}$ the region of outage due to absorption covers the whole polar cap. On $20 \mathrm{Mc} / \mathrm{s}$ only the northernmost links are out.

In figure 7 the charts for 1200 U'T September 13 are shown. At this time the ionosphere is strongly disturbed; $f_{0} F_{2}$ is well below normal over a wide region, a large area of sporadic $E$ is found over Canada, and there is enhanced absorption in the east. However, the large region of polar cap absorption is no longer found. On the $10 \mathrm{Mc} / \mathrm{s}$ chart, the available modes show several differences from 24 hours before. First, in the west there are many links on which $F$-layer support is no longer available. Secondly, there is in the west an extensive region where $\vec{E}_{s}$ modes are available. Fortunately this $E_{s}$ support comes when the $F_{2}$-layer support is weakened. Thirdly, on several links in the east there is outage due to absorption. This occurs at relatively low

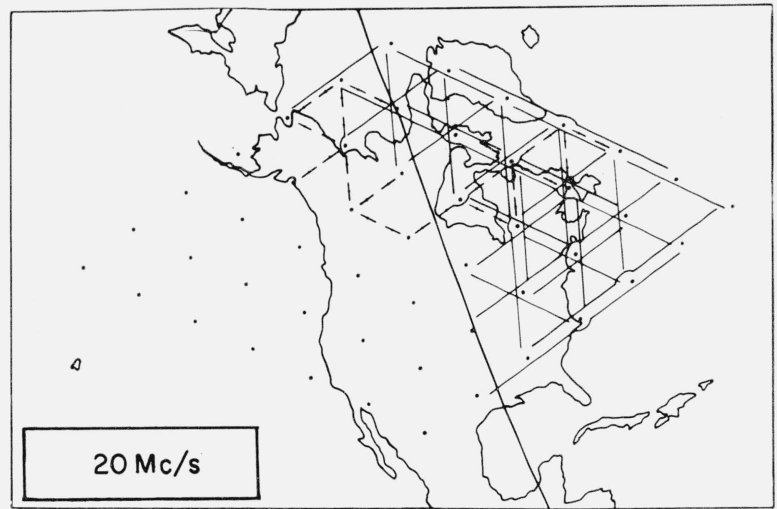

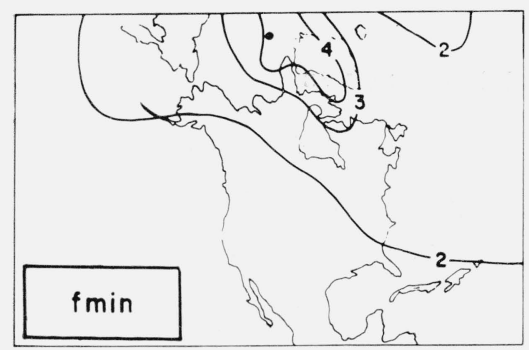

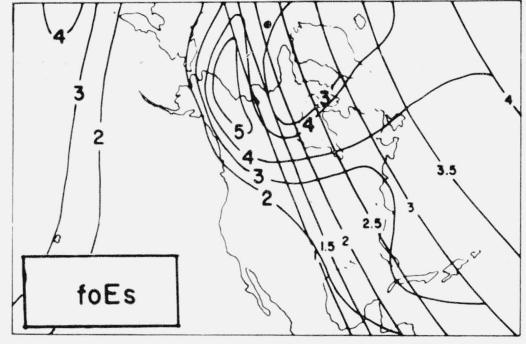

12 SEPTEMBER 1957 1200 UT

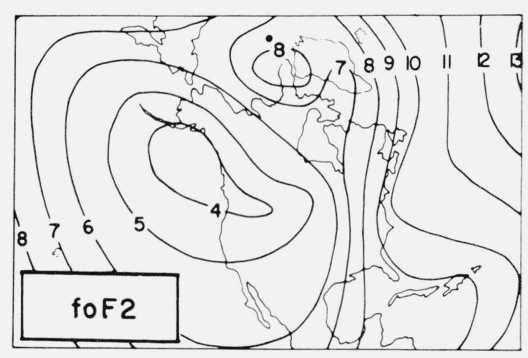

Figure 5. Communication and ionospheric charts for 1200 UT September 12, $195 \%$. 

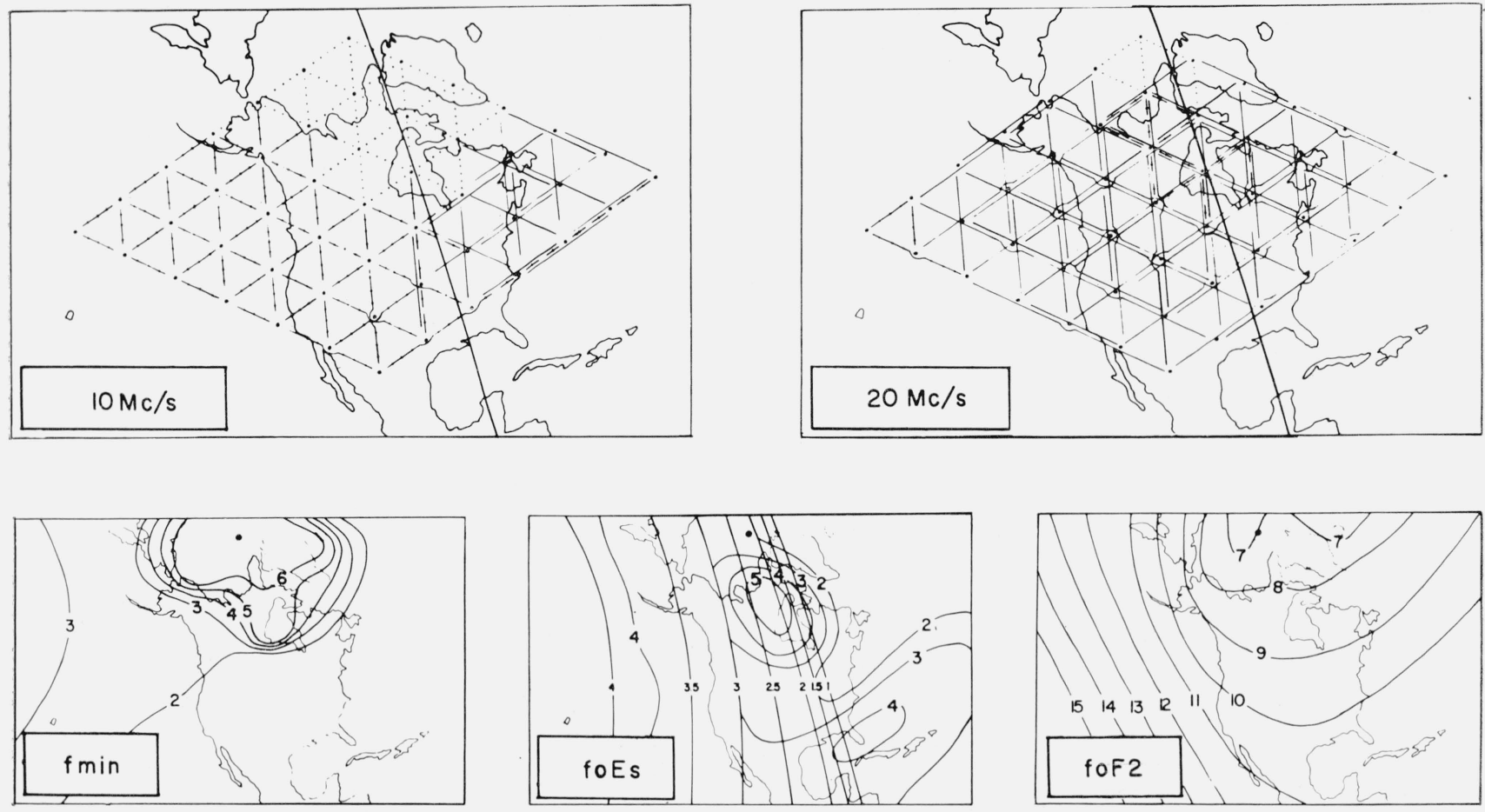

13 SEPTEMBER 1957

OOOO UT

Figure 6. Communication and ionospheric charts for 0000 UT September 13, $195 \%$.
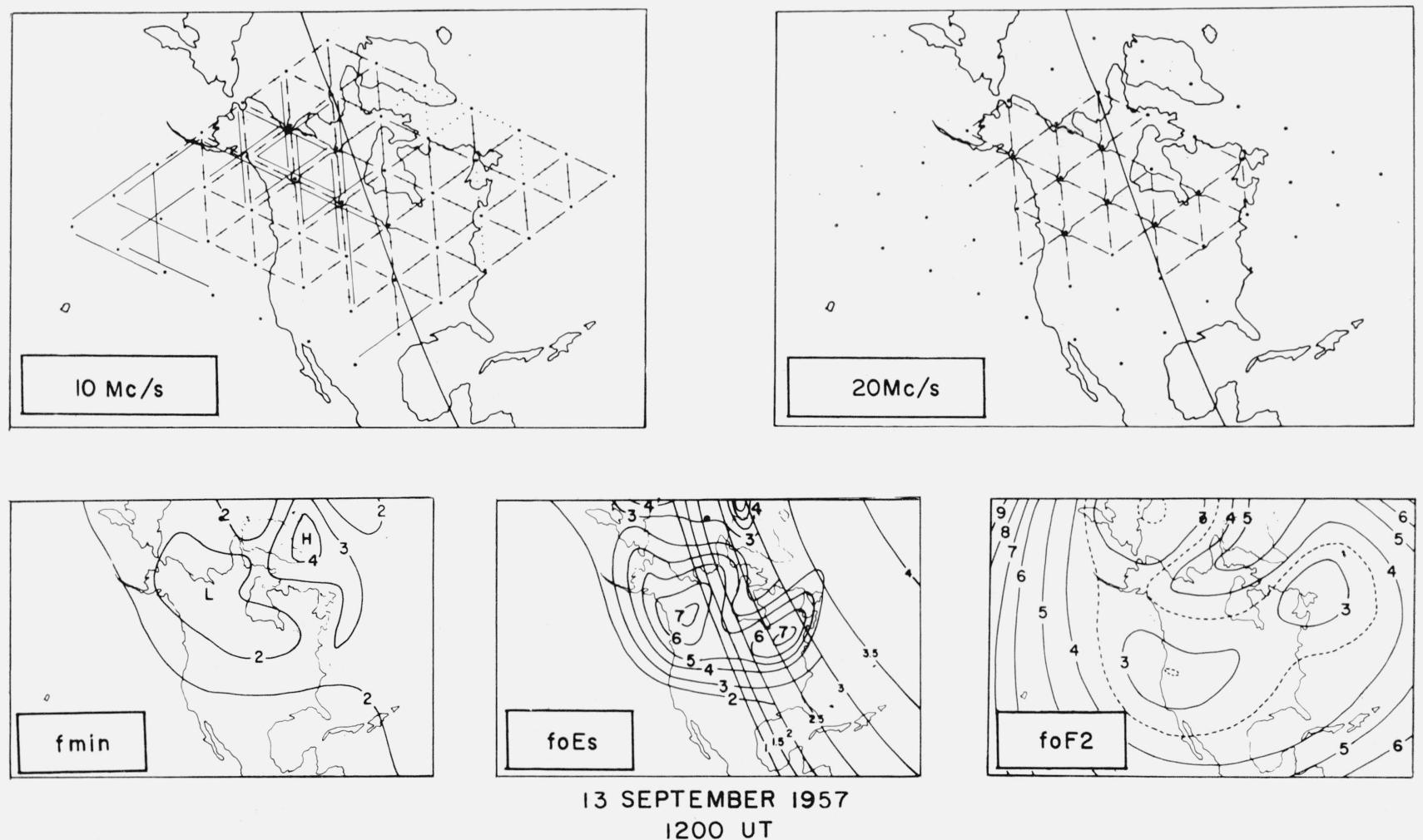

1200 UT

FIGURE 7. Communication and ionospheric charts for 1200 UT September 13, $195 \%$. 
latitudes compared to outages due to polar cap absorption. Elsewhere in the east regular E-layer support is available as usual.

On the $20 \mathrm{Mc} / \mathrm{s}$ chart, communication is quite different than on the day before. The normal $F$ layer modes in the illuminated portion of the network are absent. However, over most of Canada the $E_{s}$ mode is available. Absorption at $20 \mathrm{Mc} / \mathrm{s}$ is not intense enough at this time to cause any additional outage.

To aid the understanding of what is found on the communication charts, a summary of the available modes is given in table 2 according to the state of the ionosphere. Only the modes, or lack of modes, which are characteristic of the particular ionospheric state under consideration are given. These characteristic communication conditions are broken down into parts according to the frequency and whether the network is illuminated or not.

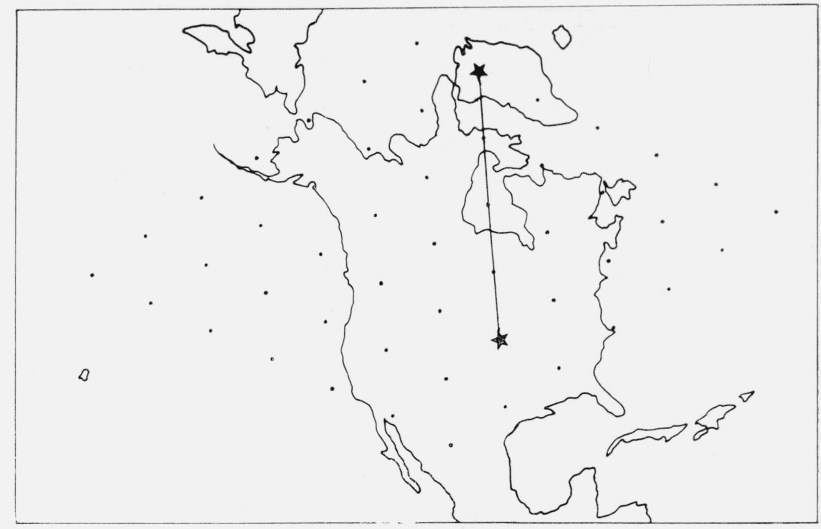

Figure 8. Map of network and hypothetical link.

TABLE 2. Main effects of the ionospheric disturbance on communication conditions over the network taken from figures 5 , 6 , and 7 , 12/1200 UT, 13/0000 UT, and 13/12000 UT

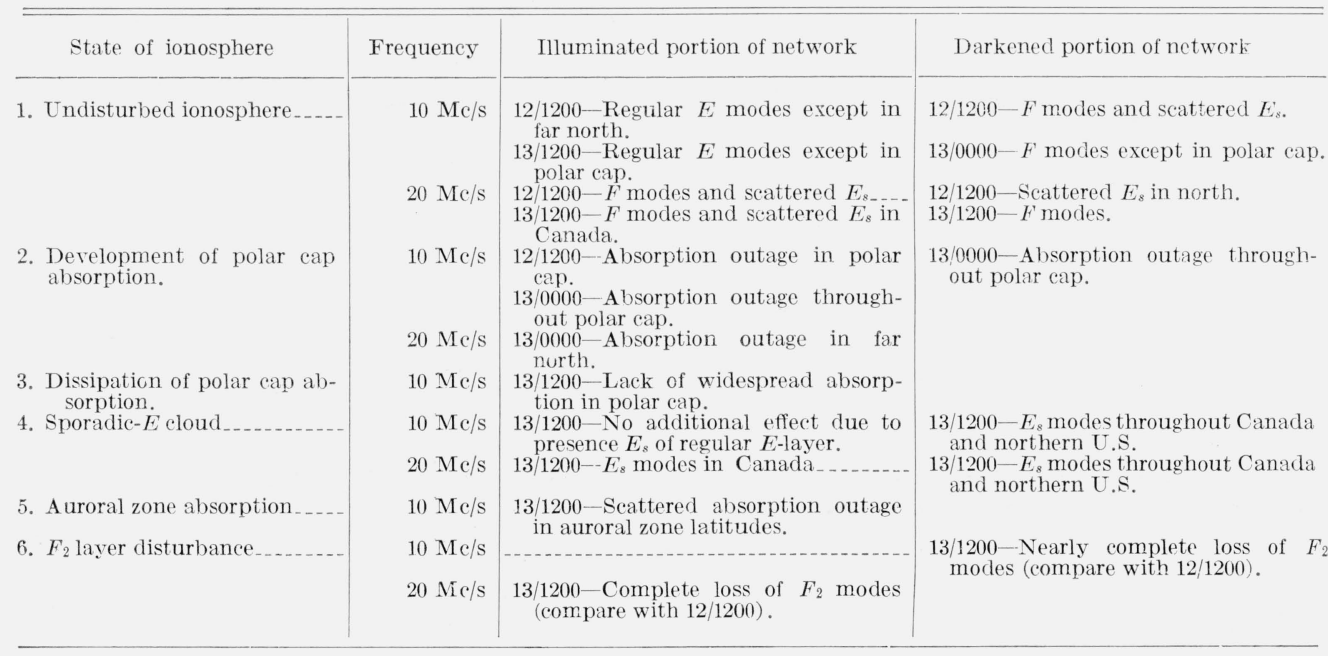

\section{Analysis of Sample Link}

To show what improvements can be achieved by the use of such communicatiou charts as described here, analysis of a particular link will be carried out. This link is a hypothetical one which has one terminal in northern Greenland and the other in central United States. These terminals have been indicated on figure 8 by a star. From the complete series of charts the frequencies available over this link have been found and are shown in figure 9 . In this figure the areas covered by heavy shading show the frequency range available for direct communication between the two terminals and the areas covered by light shading apply to indirect communication. Indirect communication may be accomplished by successive relays directly between the terminals or by relays using links away from a direct path.
On the 11 th, the frequency range available is about what would be expected for a quiet day. This range has been indicated by dashed curves for all five days so that communication on every day may be easily compared. After 1200 U'T on the 12th, normal direct communication is disrupted. The outage starts at the lower frequencies and proceeds to higher ones. Thus, the available frequencies for direct communication are reduced to a narrow band and are finally cut off altogether when the $f_{0} F_{2}$ decreases sufficiently, i.e., 2300 U'T. However, indirect communication is still available. In fact, indirect communication is available throughout the whole period of the storm. On the 13th there are occasions due to the presence of sporadic $E$, when direct communication is possible, but only over a narrow bandwidth. On the 14th, there is some recovery toward normal conditions and on the 15th there is further recovery. It should be noted that 


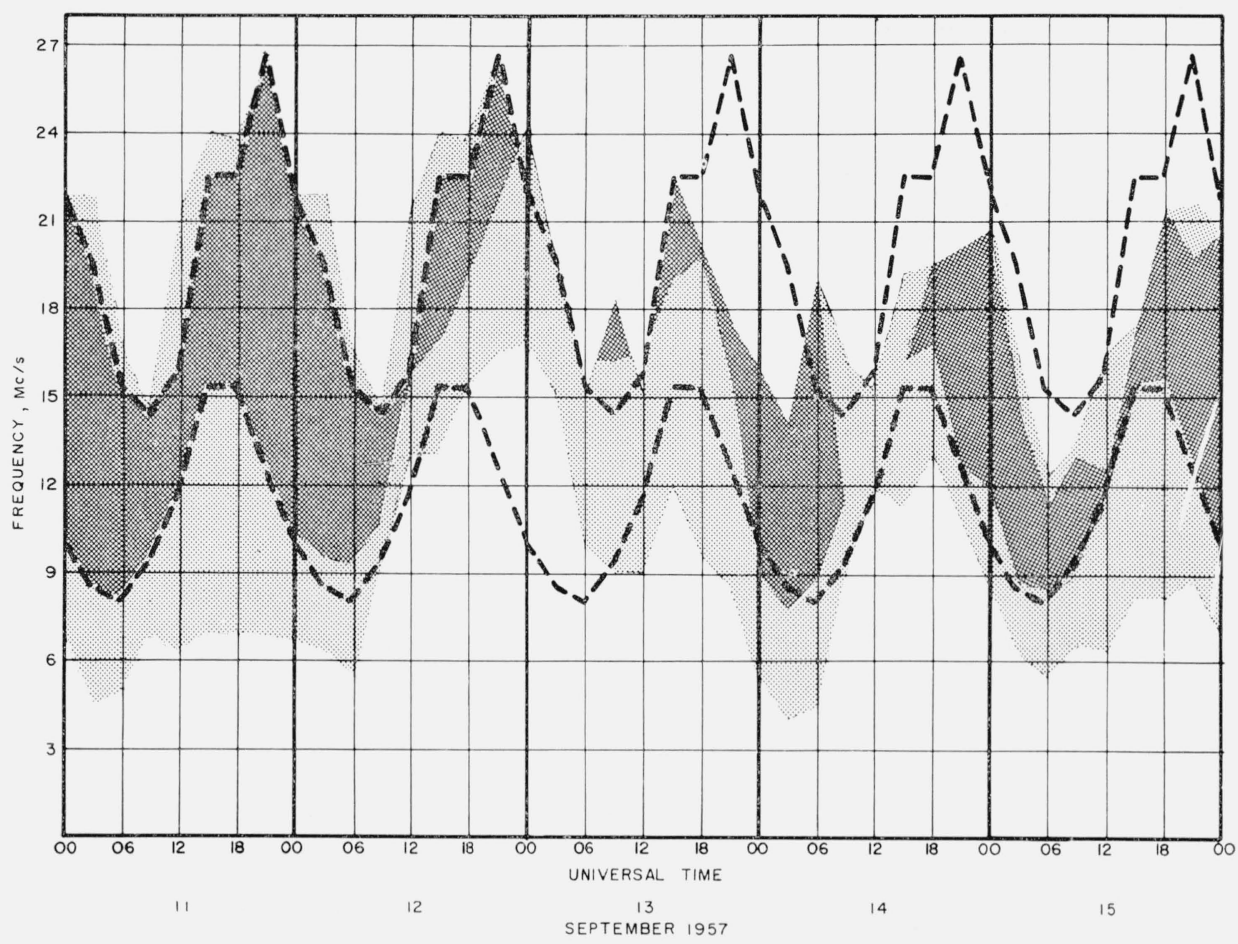

Figure 9. Frequency range available on hypothetical link.

although indirect communication is possible at all times during the five-day period on this link, it does not necessarily mean that communication on other links in the network can be maintained during the storm. However, the degree of improvement with relaying and alternate routing is considerable. It should also be noted that frequency switching at relay points has not been included in the example shown in figure 9 . In practice it is expected that one frequency may be used for initial transmission and another frequency for relay from an intermediate station. Such frequency switching will increase the range available and will add to the overall flexibility of network operations.

TABLE 3.-Hours available per day on indirect (i) and direct (d) links using communicating frequencies $3 \mathrm{Mc} / \mathrm{s}$ apart.

\begin{tabular}{|c|c|c|c|c|c|c|c|}
\hline \multirow{2}{*}{ Day } & \multicolumn{7}{|c|}{ Number of frequencies - at least: } \\
\hline & $1 \mathrm{i}$ & $2 \mathrm{i}$ & $3 \mathrm{i}$ & $1 d$ & $1 \mathrm{~d}+1 \mathrm{i}$ & $1 d+2 \mathrm{i}$ & $2 d$ \\
\hline $\begin{array}{l}11 \\
12 \\
13 \\
14 \\
15\end{array}$ & $\begin{array}{l}24 \\
24 \\
24 \\
24 \\
24\end{array}$ & $\begin{array}{l}24 \\
23 \\
23 \\
24 \\
24\end{array}$ & $\begin{array}{l}23 \\
20 \\
11 \\
16 \\
20\end{array}$ & $\begin{array}{r}24 \\
21 \\
6 \\
16 \\
20\end{array}$ & $\begin{array}{r}24 \\
20 \\
6 \\
16 \\
22\end{array}$ & $\begin{array}{r}23 \\
19 \\
6 \\
12 \\
18\end{array}$ & $\begin{array}{r}21 \\
10 \\
1 \\
12 \\
13\end{array}$ \\
\hline
\end{tabular}

To show the improvement in communication if intermediate contacts are made, a simple numerical index is used. The index is based on the number of hours per day that communication is possible on a specific number of frequencies at $3 \mathrm{Mc} / \mathrm{s}$ intervals as shown in figure 9 . In table 3 the number of hours found from figure 9 is shown by day according to the number of available frequencies. For example, at $1800 \mathrm{U}$ T on the $14 \mathrm{th}, 18 \mathrm{Mc} / \mathrm{s}$ is available for direct communication and $15 \mathrm{Mc} / \mathrm{s}$ is available for indirect communication. So this hour would count as being available under column $1 \mathrm{~d}+1 \mathrm{i}$. Also, this hour is available under columns $1 \mathrm{i}, 2 \mathrm{i}$, and $1 \mathrm{~d}$.

If only one frequency on an indirect path is required (column 1i), communication is available $24 \mathrm{hr}$ on all five days. However, if at least one frequency on a direct path is required (column 1d), communication is reduced on the $13 \mathrm{th}$, for example, to only $6 \mathrm{hr}$. It is noted that normal operations are represented by the column $1 \mathrm{~d}$. That is, communication is maintained on at least one frequency selected from several spaced $3 \mathrm{Mc} / \mathrm{s}$ apart. While the number of hours available drops to six on the 13th, using normal operations, there is no outage on any day using relays and link switching.

\section{Concurrent WWV Reception}

As a check on the validity of the results shown on the communication charts, the reception quality of WWV transmitted from Washington, D.C., is analyzed. WWV data of several Canadian stations are recorded hourly on the basis of a quality index, which consists of whole numbers $0-9$, representing no reception to excellent reception [Canadian Defense Research Board, 1957]. This quality index is dependent upon signal strength, noise level, amount of fading, and personal bias. In spite of the number of factors affecting the index, it provides a means to analyze quantitatively the reception of WWV during cbanging ionospheric conditions. The reception quality of WWV for 10 and $20 \mathrm{Mc} / \mathrm{s}$ at four Canadian stations is shown in figure 10 . 
The darkened portions of the figure represent nighttime hours at the longitude of the stations. The remaining portions represent daytime hours. The three heavy vertical lines indicate the times applicable to figures 5, 6, and 7 .

As stated before, communication on the 11th is representative of quiet ionospheric conditions. At this time, WWV reception on $20 \mathrm{Mc} / \mathrm{s}$ is low at night and high in the day, and on $10 \mathrm{Mc} / \mathrm{s}$ reception is high at night and somewhere between low and high in the day. On the 12 th, this pattern holds throughout the day on $20 \mathrm{Mc} / \mathrm{s}$, but only until about $1200 \mathrm{U}$ T on $10 \mathrm{Mc} / \mathrm{s}$ due to the presence of polar cap absorption.

On the 13th, the pattern of reception bears little resemblance to the normal one. Reception on 20 $\mathrm{Mc} / \mathrm{s}$ is enhanced intermittently during the night hours by sporadic $E$, and reduced during the daylight hours by the reduction of $f_{0} F_{2}$. On $10 \mathrm{Mc} / \mathrm{s}$, reception is somewhat reduced by the presence of auroral zone absorption. In addition, reception on $10 \mathrm{Mc} / \mathrm{s}$ fluctuates strongly. This is due to both sporadic $E$ and to the narrowness of the band of auroral zone absorption.

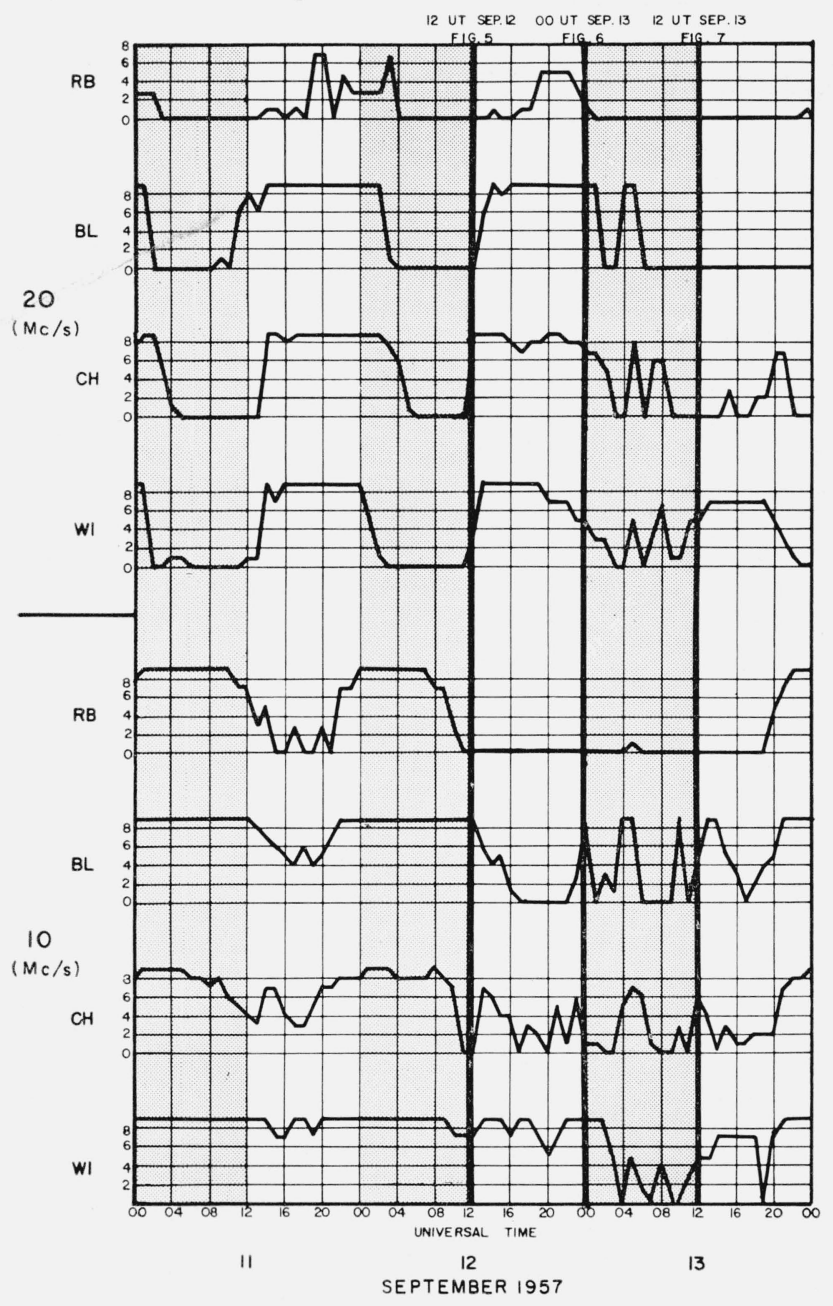

Figure 10. Reception quality index $(0-9)$ of $W W V$ for 10 and $20 \mathrm{Mc} / \mathrm{s}$ at four Canadian stations.
A comparison of the experimental data shown on figure 10 with the communication charts or with the data for the sample link shows that the results of the hypothetical network are not very different from real conditions. It should be noted that WWV reception before and after the times indicated by the heavy lines on figure 10 can be compared in a general way with propagation conditions in portions of the network other than what is found between Washington and the four receiving stations. Remembering that 15 degrees of longitude is equivalent to $1 \mathrm{hr}$, it can be seen that conditions in the western portion of the network are equivalent to what is found up to about $4 \mathrm{hr}$ before the chart time (on fig. 10) and in the eastern portion, up to about $3 \mathrm{hr}$ after the chart time. One additional point is that at these chart times the twilight zone lies just over the four receiving stations, so daytime and nighttime conditions are separated on the charts by the longitude of the stations.

\section{Conclusions}

According to the preceding results, HF signals can be continuously propagated in polar regions even during a period of severe ionospheric storminess. By a systematic analysis of ionospheric data it is possible to find the available frequencies and routes. It was found that communication between polar stations and mid-latitude stations should have been possible most of the time with frequency and link switching. It was also found that the results of the network analysis were supported by recordings of WWV transmissions. It is suggestive, at least, that the communications concept discussed here may well set the stage for establishment of a reliable globalpolar communication system.

I appreciate the helpful advice and discussion given by S. C. Coroniti and R. Penndorf. Thanks is given to Mrs. C. Kotce for her help in data reduction and computations. The assistance of Miss E. Guilbault in some of the computations and drafting work is greatly appreciated.

This work was supported by the Electronic Research Directorate, A. F. Cambridge Research Laboratories, Bedford, Mass., under contract AF19(604)4092 .

\section{References}

Bailey, D. K., Abnormal ionization in the lower ionosphere associated with cosmic-ray flux enhancements. Proc. IRE 47, 255 (1959).

Canadian Defense Research Board, Canadian ionospheric data (series), DRTE, Ottawa, Canada (1957).

Kane, J. A., Re-evaluation of ionospheric electron densities and collision frequencies derived from rocket measurements, Paper presented at AGARD meeting, Athens, Greece (1960).

Nicolet, M. and A. C. Aikin, The formation of the D region of the ionosphere, J. Geophys. Res. 65, 1469 (1960).

Rawer, K., The ionosphere, (Frederick Ungar Publ. Co., New York, 1956).

(Paper 67D1-239) 